\title{
Mortalidade em idosos com fratura de fêmur proximal em um Hospital Universitário
}

\author{
Mortality in elderly people with proximal femur fractures at a University Hospital \\ Mortalidad en ancianos con fracturas proximales de fémur en un Hospital Universitario
}

Rodrigo Santiago Moreira ${ }^{1 *}$, Jessiel Gonçalves de Souza ${ }^{1}$, Alexander Rocha Siqueira ${ }^{2}$, Mariza Dias Xavier $^{2}$, Samuel de Paiva Oliveira², Claudiana Donato Bauman².

\begin{abstract}
RESUMO
Objetivo: Analisar a relação entre o tempo de internação hospitalar e a taxa de mortalidade de pacientes idosos com fratura de fêmur proximal. Métodos: Essa pesquisa possui caráter epidemiológico, com delineamento documental, retrospectivo, descritivo e analítico, com amostra constituída por 570 pacientes que deram entrada com fratura de fêmur entre o período de janeiro de 2018 a dezembro de 2019. Utilizou-se um formulário sociodemográfico para a evolução (retrospectiva) das variáveis. Os dados foram analisados através da estatística descritiva utilizando o programa IBM SPSS Statistics. Estudo foi aprovado por Comitê de Ética em Pesquisa. Resultados: Dos 570 casos, 263 ocorreram em 2018 e 307 em 2019 . Quanto aos óbitos, 15 pacientes faleceram com idade superior a 81 anos, maioria do sexo feminino e tempo de internação maior que 15 dias. Já em 2019,11 pacientes foram a óbito, em sua maioria (63,64\%), com idade superior a 81 anos, do sexo feminino, entretanto, com tempo médio de internação de 8 a 15 dias. Conclusão: O estudo revelou que o maior tempo de internação hospitalar influencia na taxa de mortalidade de pacientes idosos com fratura de fêmur proximal aumentando o risco de óbito.
\end{abstract}

Palavras-chave: Taxa de mortalidade, Internação hospitalar, Fratura femoral.

\begin{abstract}
Objective: To analyze the relationship between the length of hospital stay and the mortality rate of elderly patients with fractures of the proximal femur. Methods: This research has an epidemiological character, with documentary, retrospective, descriptive and analytical design with a sample consisting of 570 patients who received a femoral fracture between the period of January 2018 to December 2019. A sociodemographic form was used for the evolution (retrospective) of the variables. The data were analyzed using descriptive statistics using the IBM SPSS Statistics program. The study was approved by the Research Ethics Committee. Results: Of the 570 cases, 263 occurred in 2018 and 307 in 2019. Regarding deaths, 15 patients died over 81 years of age, most of whom were female and spent more than 15 days in hospital. In 2019, 11 patients died, majority (63.64\%), also aged over 81 years, female, however, with an average length of stay from 8 to 15 days. Conclusion: The study revealed that the longer hospital stay influences the mortality rate of elderly patients with fractures of the proximal femur, increasing the risk of death.
\end{abstract}

Key words: Mortality rate, Hospitalization, Femoral fracture.

\section{RESUMEN}

Objetivo: Analizar la relación entre el tiempo de estancia hospitalaria y la tasa de mortalidad de los pacientes ancianos con fractura de fémur proximal. Métodos: Esta investigación tiene un carácter epidemiológico, con diseño documental, retrospectivo, descriptivo y analítico, con una muestra conformada por 570 pacientes que recibieron una fractura de fémur entre el período de enero de 2018 a diciembre de 2019 . Se utilizó un formulario sociodemográfico para la evolución (retrospectiva) de las variables. Los datos se analizaron mediante estadística descriptiva utilizando el programa IBM SPSS Statistics. El estudio fue aprobado por el Comité de Ética en Investigación. Resultados: De los 570 casos, 263 ocurrieron en 2018 y 307 en 2019 . En cuanto a las defunciones, fallecieron 15 pacientes mayores de 81 años, la mayoría mujeres y estadía hospitalaria superior a 15 días. En 2019 fallecieron 11 pacientes, en su mayoría (63,64\%), mayores de 81 años, sin embargo mujeres, con una estancia hospitalaria media de 8 a 15 días. Conclusión: El estudio reveló que la mayor estancia hospitalaria influye en la tasa de mortalidad de los pacientes ancianos con fracturas de fémur proximal, aumentando el riesgo de muerte.

Palabras clave: Tasa de mortalidad, Hospitalización, Fractura femoral.

\footnotetext{
${ }^{1}$ Hospital Universitário Clemente de Faria (HUCF), Montes Claros - MG. *E-mail: rodrigosantiago32@gmail.com

2 Universidade Estadual de Montes Claros (Unimontes), Montes Claros - MG.
} 


\section{INTRODUÇÃO}

O envelhecimento é um processo natural e inerente a todo ser humano, e acompanhado de uma série de alterações, sendo elas morfológicas, bioquímicas, funcionais e psicológicas. Essas modificações geram uma vulnerabilidade e uma maior incidência nos processos patológicos nos idosos, interferindo diretamente na qualidade de vida e aumento da mortalidade nessa população (FILHO IP, et al., 2019).

De acordo com a Organização Mundial da Saúde (OMS), o idoso é definido como todo indivíduo com a faixa etária acima de 60 anos de idade. Com a evolução nas políticas públicas mundiais de saúde, a expectativa de vida a nível global vem aumentando progressivamente. Com isso, o número de traumas no idoso segue a mesma tendência e apresenta acréscimos significativos, sendo a fratura de fêmur proximal uma das consequências mais temidas e mais frequente da queda no idoso. Esse tipo de injúria traz muitos malefícios ao paciente, com elevadas taxas de morbidade e mortalidade, sendo constituído um sério problema de saúde pública (PINTO JA, et al., 2019; WHO, 2017, SILVA NTF, et al., 2018).

Segundo a estimativa do Instituto Brasileiro de Geografia e Estatística (IBGE), a projeção para o ano de 2018, foi de cerca de 28 milhões de idosos, representando uma porcentagem de $13 \%$ da população do país, em relação as outras faixas etárias. A projeção da população atualizada no ano de 2018 estimou que em 2043, um quarto da população deverá ter mais de 60 anos, enquanto os jovens representarão apenas 16,4\%. Comparado a outros países como por exemplo, a França, o Brasil, a China e a Índia terão apenas cerca de 20 anos para adaptar a essas mudanças, que já ocorrem há algum tempo (IBGE, 2010; LINHARES JE, et al., 2019).

A população idosa vem apresentando aumento significativo desde o ano de 1940 e fatores internos e externos podem estar diretamente relacionados. Hábito alimentar, sedentarismo, prática de atividade física, avanço da ciência, entre outros fatores são considerados como eventos que influenciam negativamente ou positivamente no processo de senescência e senilidade, uma vez, que a preservação da qualidade de vida na velhice é o principal desafio imposto pela longevidade dos idosos, principalmente conectados à preservação das atividades da vida diária e o aumento da sobrevida (SILVA KR, et al., 2020; MARTINS NPR, et al., 2020; SOUZA FJM, et al., 2020).

Os traumas em idosos que resultam em fraturas de fêmur proximal geralmente são de baixa energia e relacionam-se às características próprias do idoso, como osteoporose, desnutrição, diminuição da acuidade visual, prejuízo das funções cognitivas, fragilidade óssea e sarcopenia. Entre essas fraturas, estão presentes as do colo femoral, transtrocantéricas e subtrocantéricas (FILHO IP, et al., 2019; PETROS RSB, et al., 2017).

As consequências das fraturas de fêmur proximal nos idosos podem ser devastadoras, alterando o equilíbrio físico, mental, funcional e social. Além disso, acarreta danos familiares, uma vez que é criada uma dependência por perda de autonomia após o trauma, gerando problemas internos. A fragilidade e o equilíbrio são itens mensuráveis que tendem a drasticamente afetarem a vida do idoso, principalmente nos casos após o trauma, onde pode haver novamente o risco de queda, o aumento de hospitalizações e o risco de morte (FILHO IP, et al., 2019; SILVA VF, et al., 2020).

De acordo com o que vem sendo discutido em relação às fraturas de fêmur, nos últimos anos tem merecido extrema atenção das autoridades sanitárias brasileiras pelo seu evidente impacto na saúde dos idosos e por suas consequências para o setor público. Estudos atuais revelam que a expectativa de vida dos pacientes que sofrem esse tipo de fratura é reduzida em 15 a 20\%, com as taxas de mortalidade relacionadas a esse agravo variando de 15 a 50\% no primeiro ano, além de aumentar o risco de mortalidade por internação, já que o ambiente contém muitos fatores que podem agravar o quadro (SOARES DS, et al., 2014).

Na maioria dos casos de fratura em idosos, há indicação cirúrgica, sendo que o tratamento conservador é escolhido em casos selecionados, como: fraturas incompletas e sem desvio ou quando o paciente não possui condições clínicas compatíveis com a abordagem cirúrgica. O tempo considerado ideal para essa abordagem varia entre 24 e 48 horas após o trauma, que é estimado dependendo do estado geral de saúde do paciente. As cirurgias feitas após 48 horas ou mais aumentam o risco de mortalidade nos primeiros 30 dias e até um ano (GUERRA TEM, et al., 2017). 
De acordo com contexto da ocorrência de traumas em idosos e o risco de mortalidade devido à fragilidade imposta pelo processo de envelhecimento, o presente estudo tem como objetivo analisar a relação entre o tempo de internação hospitalar e a taxa de mortalidade de pacientes idosos com fratura de fêmur proximal.

\section{MÉTODOS}

Essa pesquisa possui caráter epidemiológico, com delineamento documental, retrospectivo, descritivo e analítico que foi realizada em um hospital do Norte de Minas Gerais, o qual se realiza atividades de ensino, pesquisa e extensão se destacando na prestação de serviços na área da saúde $100 \%$ assistido pelo Sistema Único de Saúde (SUS). Na área de Urgência e Emergência, o hospital é classificado como trauma nível dois, contando com um pronto socorro 24 horas, e a Ortopedia como uma das especialidades.

A amostra desse estudo foi constituída por 570 pacientes que deram entrada no Hospital Universitário Clemente de Faria com fratura de fêmur entre o período de janeiro de 2018 a dezembro de 2019, com informações provenientes do sistema de informação. Os pesquisadores foram devidamente calibrados e realizaram a extração dos dados, por meio da análise dos prontuários e protocolos de coleta préestabelecidos.

Os critérios de inclusão se relacionaram a pacientes de ambos os sexos, com idade a partir de 60 anos com fratura fêmur. Foram excluídos prontuários com dados incompletos. Utilizou-se um formulário sociodemográfico para a evolução (retrospectiva) das variáveis.

Os dados foram analisados através da estatística descritiva (frequência simples e relativa), utilizando o programa Statical Package for Social Science (SPSS), versão 20.0. O projeto desse estudo foi submetido à apreciação do Comitê de Ética em Pesquisa da Universidade Estadual de Montes Claros (UNIMONTES), tendo sido aprovado por meio do parecer consubstanciado 4.214.370. Essa pesquisa dispensou a utilização do Termo de Consentimento Livre e Esclarecido devido os dados serem de obtenção secundária retrospectiva de pacientes que foram atendidos ou que vieram a óbito, respeitando as diretrizes da resolução $\mathrm{CNS} \mathrm{N}^{\circ} 466$ de 2012.

\section{RESULTADOS}

Foram investigados os casos de idosos internados com diagnóstico de fratura de fêmur proximal dos anos de janeiro de 2018 a dezembro de 2019. No total, foram 570 casos, sendo 263 do ano de 2018 e 307 do ano de 2019. Como descrito na Tabela 1, o tipo de fratura com maior ocorrência foi a Pertrocantérica tanto no ano de 2018 (119 casos) quanto no ano de 2019 (163 casos), em segundo lugar, foi a de colo de fêmur, com 116 (2018) e 118 (2019) casos. Os outros casos foram de fratura subtrocantéricas (Tabela 1).

Tabela 1 - Número de idosos internados por tipo de fratura nos anos de 2018 e 2019.

\begin{tabular}{lcccc}
\hline \multirow{2}{*}{ Tipos de fraturas } & \multicolumn{2}{c}{$\mathbf{2 0 1 8}$} & $\mathbf{n}$ & $\mathbf{2 0 1 9}$ \\
\cline { 2 - 5 } & $\mathbf{n}$ & $\mathbf{\%}$ & 26 & $\%$ \\
\hline Subtrocantérica & 28 & 10,65 & 163 & 53,46 \\
Pertrocantérica & 119 & 45,25 & 118 & 38,44 \\
Colo do fêmur & 116 & 44,10 & $\mathbf{3 0 7}$ & $\mathbf{1 0 0}$ \\
\hline Total & $\mathbf{2 6 3}$ & $\mathbf{1 0 0}$ &
\end{tabular}

Fonte: Moreira RS, et al., 2020. Dados extraídos do sistema de informação.

É possível observar que em comparativo dos anos 2018 e 2019 de acordo com a Tabela 1, o ano de 2018 teve menos atendimentos de fraturas em idosos, entretanto, foi o ano que teve o maior número de óbitos, sendo que no outro ano ocorreram mais atendimentos e menos óbitos.

Na Tabela 2 será apresentada a relação de idosos que vieram a óbito de acordo com o número de pacientes que deram entrada com diagnóstico de fratura. No ano de 2018 foi a óbito 15 pacientes dos 263 que deram entrada na ortopedia. Já em 2019, 11 pacientes dos 307 vieram a óbito (Tabela 2). 
Tabela 2 - Relação entre número de idosos vivos e que vieram a óbito por ano.

\begin{tabular}{lcc}
\hline \multirow{2}{*}{ Ano } & Vivos & Óbitos \\
\cline { 2 - 3 } & $\mathbf{n}$ & $\mathbf{n}$ \\
\hline $\mathbf{2 0 1 8}$ & 263 & 15 \\
$\mathbf{2 0 1 9}$ & 307 & 11
\end{tabular}

Fonte: Moreira RS, et al., 2020. Dados extraídos do sistema de informação.

Os dados apresentados na Tabela 3 revelaram que a maioria dos idosos que vieram à óbito em 2018 possuíam idade superior a 81 anos $(73,34 \%)$ e nove $(60 \%)$ eram do sexo feminino. Já em relação ao tempo de internação, nove (60\%) ficaram internados mais de 15 dias, sendo que o setor final (unidade de internação) da maioria dos pacientes $(53,34 \%)$ foi pela cirurgia geral (Tabela 3$)$.

Tabela 3 - Variáveis de estudo entre os óbitos do ano de 2018.

\begin{tabular}{|c|c|c|}
\hline Variável & $\mathbf{n}$ & $\%$ \\
\hline \multicolumn{3}{|l|}{ Idade } \\
\hline $60-70$ anos & 2 & 13,33 \\
\hline $71-80$ anos & 2 & 13,33 \\
\hline Acima 81 anos & 11 & 73,34 \\
\hline \multicolumn{3}{|l|}{ Sexo } \\
\hline Feminino & 9 & 60,0 \\
\hline Masculino & 6 & 40,0 \\
\hline \multicolumn{3}{|c|}{ Tempo de internação } \\
\hline Até 7 dias & 3 & 20,0 \\
\hline 8-15 dias & 3 & 20,0 \\
\hline Mais de 15 dias & 9 & 60,0 \\
\hline \multicolumn{3}{|c|}{ Unidade final de Internação } \\
\hline Ortopedia & 0 & 0,0 \\
\hline UTI & 4 & 26,66 \\
\hline Cirurgia Geral & 8 & 53,34 \\
\hline Pronto Socorro & 3 & 20,0 \\
\hline
\end{tabular}

Fonte: Moreira RS, et al., 2020. Dados extraídos do sistema de informação.

No ano de 2019, como apresentado na Tabela 4, foi possível evidenciar que sete $(63,64 \%)$ dos idosos tinham acima de 81 anos de idade e que nove $(81,82 \%)$ eram do sexo feminino. Em relação ao tipo de fratura mais frequente entre os óbitos foi a pertrocantérica com $54,55 \%$. Em relação ao tempo de internação $45,45 \%$ permaneceram no hospital entre oito a 15 dias. A unidade final de internação no caso dos óbitos de 2019 foi 
a unidade de terapia intensiva (63,64\%). As variáveis relacionadas quanto aos óbitos no ano de 2019 foram a idade, sexo, tempo de internação e unidade final de internação (Tabela 4).

Tabela 4 - Variáveis de estudo entre os óbitos do ano de 2019.

\begin{tabular}{|c|c|c|}
\hline Variável & $\mathbf{n}$ & $\%$ \\
\hline \multicolumn{3}{|l|}{ Idade } \\
\hline $60-70$ anos & 0 & 0,0 \\
\hline $71-80$ anos & 4 & 36,36 \\
\hline Acima 81 anos & 7 & 63,64 \\
\hline \multicolumn{3}{|l|}{ Sexo } \\
\hline Feminino & 9 & 81,82 \\
\hline Masculino & 2 & 18,19 \\
\hline \multicolumn{3}{|l|}{ Tipo de fratura } \\
\hline Subtrocantérica & 0 & 0,0 \\
\hline Pertrocantérica & 6 & 54,55 \\
\hline Colo do fêmur & 5 & 45,45 \\
\hline \multicolumn{3}{|c|}{ Tempo de internação } \\
\hline Até 7 dias & 2 & 18,19 \\
\hline 8-15 dias & 5 & 45,45 \\
\hline Mais de 15 dias & 4 & 36,36 \\
\hline \multicolumn{3}{|c|}{ Unidade final de Internação } \\
\hline Ortopedia & 0 & 0,0 \\
\hline UTI & 7 & 63,64 \\
\hline Cirurgia Geral & 4 & 36,36 \\
\hline Pronto Socorro & 0 & 0,0 \\
\hline
\end{tabular}

Fonte: Moreira RS, et al., 2020. Dados extraídos do sistema de informação.

\section{DISCUSSÃO}

De acordo com os resultados apresentados anteriormente, nos anos de 2018 e 2019, a fratura pertrocantérica foi a de maior ocorrência, evidenciada tanto nos casos que deram entrada no hospital investigado, como nos casos que vieram a óbito.

A mortalidade decorrente das fraturas de fêmur proximal representa um ponto importante, pois é a principal causa de morte por trauma em pessoas com mais de 75 anos de idade. Alguns estudos apontaram que até $50 \%$ dos pacientes evoluíram à óbito dentro dos primeiros 6 meses após o trauma. Outros dados epidemiológicos demostraram que um ano após a fratura de fêmur proximal a mortalidade girava em torno de 14 a $36 \%$, sendo que apenas 50 a $65 \%$ dos pacientes possuíam uma recuperação completa da atividade funcional (PETROS RSB, et al., 2017; GUERRA MTE, et al., 2017).

Estudo realizado por Soares DS, et al. (2015), do tipo caso-controle (um grupo de casos e dois grupos controle), com indivíduos de idade acima de 60 anos, residentes no município de São Sebastião do ParaísoMG, no período de 2005 a 2012, evidencia que os principais fatores de risco de fraturas em idosos que 
consequentemente leva a internação, na maioria dos casos, são: idade, sexo, sedentarismo, osteoporose, incapacidade física prévia, menopausa precoce e presença de comorbidades. A osteoporose, mais especificamente, é considerada o principal fator de risco para aumento na incidência de fratura em idosos acima de 60 anos de idade, principalmente em mulheres. Outro fator importante também analisado na pesquisa foi que idosos portadores de Hipertensão Arterial Sistêmica em uso de Anti-hipertensivos foram mais propensos a sofrer de fêmur.

Foi evidenciado que os casos de óbitos ocorreram em maior escala em pacientes com idade superior a 81 anos e que a maioria eram mulheres. Os fatores considerados determinantes para a mortalidade desses pacientes, como descrito em outros estudos, foram: a idade avançada, o estado mental, o estado físico, o sexo masculino, capacidade de mobilidade antes da fratura, comorbidades prévias e o atraso no tratamento. A identificação precoce desses fatores determinantes poderá auxiliar na diminuição dos índices de mortalidade (FILHO JA, et al., 2019; PAULA FL, 2014).

Em um estudo realizado por Manoli A, et al. (2017), foi verificado que pacientes idosos possuíam uma taxa de mortalidade hospitalar significativamente maior do que as populações mais jovens, entretanto, foi evidenciado uma maior ocorrência entre o sexo masculino do que feminino. Isso provavelmente decorre das múltiplas comorbidades que os idosos apresentam e do estado imunológico mais suprimido.

No estudo realizado por Conceição AM, et al. (2016), com objetivo descrever o perfil epidemiológico dos internados com fraturas de fêmur em Salvador, Bahia, no período de 2003 a 2012, identificou 10.721 internações e 195 óbitos hospitalares. Foram mais acometidos os indivíduos de 20-49 anos e maiores de 70 anos, do sexo masculino, com índice de maior mortalidade hospitalar entre as mulheres sendo que o tempo médio de permanência foi 12,2 dias. O estudo supracitado está em conformidade com esse trabalho, uma vez que houve maior mortalidade no sexo feminino.

Em uma pesquisa realizada por Soares DS, et al. (2014), que objetivou avaliar os dados secundários registrados no SIH-SUS sobre a distribuição das fraturas de fêmur ocorridas no Brasil, no período de 2008 a 2012, teve como resultados a ocorrência de mais de 181 mil casos de fraturas de fêmur em pessoas com idade maior ou igual a 60 anos em todo o país gerando gastos em internações para os cofres públicos. Evidenciou também a alta morbidade e mortalidade na população idosa, sendo as fraturas de fêmur responsáveis por significativa repercussão econômica e social de relevância para o Brasil. Foi identificado também que dos mais de 181 mil casos de fraturas de fêmur identificados neste estudo, 67,5\% ocorreram em mulheres, o que também foi descrito por outros estudos presente nessa pesquisa e corrobora para os dados achados, uma vez que houve maior incidência no sexo feminino.

A questão da evidente suscetibilidade do gênero feminino, como mostrado em muitos estudos, chama a atenção para o problema da osteoporose, uma doença sistêmico-progressiva caracterizada pela diminuição da massa óssea, que pode ser devido a diversos fatores, entre eles a própria questão hormonal vivenciada pelas mulheres no período da menopausa. Nesse período ocorre uma série de distúrbios hormonais, impactando diretamente sobre a qualidade óssea (SOARES DS, et al., 2014; CONCEIÇÃO AM, et al., 2016).

O tempo de internação dos indivíduos apresentaram dados relevantes, uma vez, que a taxa de mortalidade foi maior entre os idosos que ficaram mais tempo internados. No ano de 2018 , a taxa de internação foi maior entre os idosos que ficaram mais de 15 dias, porém, em 2019, foi maior entre os que permaneceram entre os que estiveram internados durante 8 a 15 dias. Vários fatores podem estar associados entre essa relação da taxa de mortalidade e o tempo de internação. Em um estudo conduzido por Pinto IP, et al. (2019), foi apresentado uma redução da taxa de mortalidade quando o paciente realiza cirurgia e recebe alta hospitalar em menos de 48 horas que deu entrada no hospital. Esses resultados corroboram com o estudo em relação ao tempo de internação, apontando fator devido à maior a exposição ao ambiente hospitalar, o que traz consigo diversas infecções.

Uma pesquisa realizada por Alcantara C, et al. (2020), com objetivo de analisar os fatores que interferem no desfecho de internação de idosos com fratura de femur no período de 2015 a 2017, no Norte do Paraná, evidenciou que, dos 189 prontuários analisados, 132 (69,9\%) idosos desenvolveram morbidades no ambiente hospitalar, sendo que a ocorrência de infecção urinária, sepse, pneumonia, lesão por pressão e a presença de duas ou mais comorbidades foram associadas ao desfecho de óbito e ao tempo de hospitalização superior 
a 10 dias. Também foi identificado que a maioria dos idosos que deram entrada na internação com fratura, os que tinham idade superior a 80 anos apresentaram maiores morbidades, sendo projetado para o sexo feminino e que a maioria desses idosos já apresentavam presença de morbidades como Hipertensão e Diabetes, podendo levar a um risco alto de óbito juntamente com um conjunto de comorbidades.

Existem vários fatores associados que contribuem para o aumento das taxas de mortalidade durante 0 tempo de permanência hospitalar, sendo as doenças já existentes que surgem como fator de risco, o número de comorbidades ou adquiridas também influencia significamente 0 desfecho da hospitalização, principalmente após o procedimento cirúrgico. Pacientes que são internados com fraturas e outras comorbidades como infecção do trato urinário ou pneumonia têm prolongamento das internações e maior incidência de complicações com risco de morte aumentado (ASTUR DC, et al., 2011).

Embora a pesquisa publicada por Franco LG, et al. (2016) destacou que o tempo de espera para cirurgia e o tempo de internação não apresentaram associação com o desfecho da mortalidade intra-hospitalar, os mesmos enfatizaram a importância da elaboração de protocolos para um melhor manejo desses pacientes. Dessa forma, os pacientes serão melhor acolhidos dentro do ambiente hospitalar e terão um tratamento mais precoce.

Em uma pesquisa realizada por Etxebarria-Foronda I, et al. (2013), com 1856 procedimentos cirúrgicos, foi destacado que a permanência hospitalar antes da cirurgia em pacientes com fratura de quadril (apesar de ainda ser um desafio devido diversos fatores), não foi encontrado associação em relação ao tempo de permanência pré-operatória intra-hospitalar e a taxa de mortalidade.

Já o estudo realizado por Rosso F, et al. (2016), composto por 1448 pacientes em um Departamento de Ortopedia, ressaltou que ao avaliar a relação entre a taxa de mortalidade em diferentes tempos de acompanhamento (30 dias, seis meses e um ano), foi identificado que a realização da cirurgia dentro de 48 horas pode estar associada a uma diminuição na taxa de mortalidade, assim como evidenciaram que o tempo de internação se mostrou fator determinante na taxa de mortalidade, corroborando com os achados do presente estudo, uma vez que os pacientes que vieram a óbito ficaram mais tempo internados no hospital.

A bibliografia especializada também aponta alguns fatores relacionados a desfechos desfavoráveis em pacientes idosos com fratura após a cirurgia, como: incapacidade prévia à fratura, deficiências cognitivas, ocorrência de uma segunda fratura associada, baixo nível funcional na data da alta e falta de vitamina $D$, além do óbito. Grande parte dos pacientes submetidos às abordagens cirúrgicas já possuem uma série de comorbidades pré-operatórias que elevam as taxas de complicações durante 0 ato cirúrgico e as taxas de mortalidade (CHOU SE, et al., 2019; FILHO JA, et al., 2019).

O estudo realizado por Smith $\mathrm{T}$, et al. (2014), que teve como objetivo, identificar características préoperatórias que estão associadas a um aumento do risco de mortalidade após a cirurgia de fratura de quadril, identificou quatro características principais associadas ao risco de mortalidade: o ECG anormal, comprometimento cognitivo, idade maior que 85 anos e mobilidade pré-fratura. Esse estudo corrobora com a maioria dos trabalhos encontrados, evidenciando a importância da relação entre a idade do paciente e a mortalidade.

A característica do ato cirúrgico é de extrema importância para a qualidade do pós-operatório e da qualidade de vida. A não ocorrência de quadros infecciosos pós-cirurgia é um grande desafio, principalmente para evitar desfechos desfavoráveis e agravos de idosos internados. Esses fatores são imprescindíveis para que possa evitar casos de óbitos, e podem ser prevenidos com a utilização de antibioticoprofilaxia de amplo espectro após o ato cirúrgico (ALCANTARA C, et al., 2020).

O local final de internação do paciente no momento do óbito não foi considerado proeminente para fins dessa investigação, uma vez que não foram avaliados os motivos para transferência. A principal limitação do presente estudo se referiu à dificuldade da coleta de dados relacionando outras variáveis, devido à ausência de algumas informações no prontuário, o que pode ter subestimado a real condição clínica do paciente. Entre as variáveis importantes que não no constavam prontuário, cita-se: comorbidades prévias dos pacientes, complicações pós-cirúrgicas, tipo de procedimento realizado e se foi realizada ou não a antibioticoprofilaxia. Outro apontamento trata-se da questão observacional, uma vez, que não é possível à afirmação de causalidade. 


\section{CONCLUSÃO}

O presente estudo revelou que o maior tempo de internação hospitalar influencia na taxa de mortalidade de pacientes idosos com fratura de fêmur proximal, além de aumentar o risco de óbito. Destaca-se maior mortalidade entre aqueles com idade superior a 81 anos e aqueles do sexo feminino. Esses achados apontam e aumentam a importância de que medidas hospitalares e de saúde pública devem ser reafirmadas para a diminuição desses fatores. Observa-se a necessidade de construção de protocolos específicos para atendimento de idosos nos ambientes hospitalares, para diminuir os índices de mortalidade e melhorar a qualidade de vida dos pacientes.

\section{REFERÊNCIAS}

1. ALCANTARA C, et al. Fatores associados ao desfecho da hospitalização de idosos submetidos a correção de fratura de fêmur. Cogitare enferm. 2020; 25.

2. ASTUR DC, et al. Fraturas da extremidade proximal do fêmur tratadas no Hospital São Paulo/Unifesp: estudo epidemiológico. RBM rev. bras. Med, 2011; 68(4,n.esp).

3. CHOU SE, et al. Risk factors and complications contributing to mortality in elderly patients with fall-induced femoral fracture: A cross-sectional analysis based on trauma registry data of 2,407 patients. Int J Surg, 2019; 66: 48-52.

4. CONCEIÇÃO AM, et al. Internações por fraturas de fêmur em salvador, bahia. Rev. baiana saúde pública, 2016; 40(2).

5. ETXEBARRIA- FORONDA I, et al. Cost and mortality associated to the surgical delay of patients with a hip fracture. Rev Esp Salud Publica. 2013; 87(6): 639-49.

6. FRANCO LG, et al. Fatores associados à mortalidade em idosos hospitalizados por fraturas de fêmur. Rev. bras. ortop., São Paulo, 2016; 51(5): 509-514.

7. FILHO JA, et al. Predictive Factors of Death after Surgery for Treatment of Proximal Femoral Fracture. Rev. bras. Ortop, 2019; 54(4): 402-407.

8. GOMES LSM. Diagnóstico precoce da infecção articular periprotética do quadril - situação atual, avanços e perspectivas. Rev. bras. ortop., São Paulo, 2019; 54(4): 368-376.

9. GUERRA MTE, et al. Mortalidade em um ano de pacientes idosos com fratura do quadril tratados cirurgicamente num hospital do Sul do Brasil. Rev. bras. ortop., São Paulo, 2017; 52(1): 17-23.

10. LINHARES JE, et al. Capacidade para o trabalho e envelhecimento funcional: análise Sistêmica da Literatura utilizando o PROKNOW-C (Knowledge Development Process - Constructivist). Ciência \& Saúde Coletiva, 2019; 24(1):53-66.

11. MANOLI A, et al. Short-Term Outcomes Following Hip Fractures in Patients at Least 100 Years Old. J Bone Joint Surg Am. 2019; 99(13): e68.

12. MARTINS NPR, et al. Quality of life of older adults admitted to a Medical Clinic Unit of a Public Hospital in Brazil. Rev Esc Enferm USP. 2020.

13. Ministério do Planejamento, Orçamento e Gestão (BR), Instituto Brasileiro de Geografia e Estatística. Indicadores Sociodemográficos e de Saúde no Brasil 2009 [Internet]. Acesso em 18 de outubro de 2020. Disponível em: <https://www.ibge.gov.br> PAULA FL. Readmissão hospitalar de idosos após internação por fratura proximal do fêmur no município do rio de janeiro. Fiocruz, 2014; Janeiro.

14. PETROS RSB, et al. Influência das fraturas do fêmur proximal na autonomia e mortalidade dos pacientes idosos submetidos a osteossíntese com haste cefalomedular. Rev. bras. ortop., São Paulo, 2019; 52(1): 57-62.

15. PINTO IP, et al. Does Early Surgical Fixation of Proximal Femoral Fractures in Elderly Patients Affect Mortality Rates? Rev. bras. Ortop. 2019; 54(4): 392-395.

16. ROSSO F, et al. Prognostic factors for mortality after hip fracture: operation within 48hours is mandatory. Injury. 2016;47 Suppl 4:S91-7.

17. SMITH T, et al. Pre- operative indicators for mortality following hip fracture surgery: a systematic review and metaanalysis. Age Ageing. 2014; 43(4):464-71.

18. SILVA NTF, et al. Profile of older adult victims of trauma cared for in the emergency care unit of a teaching. Ciênc Cuid Saúde. 2018; 7(2):1-8.

19. SILVA KR da, et al. Perfil epidemiológico de pacientes idosos atendidos em um pronto socorro. Rev enferm UFPE on line. 2020.

20. SILVA VF, et al. Estimulação cerebral não invasiva e efeito sinérgico do exercício físico sobre a fragilidade e equilíbrio de idosos. Revista Eletrônica Acervo Saúde (REAS), 2020; 12(4): e2602.

21. SOARES DS, et al. Análise dos fatores associados a quedas com fratura de fêmur em idosos: um estudo caso-controle. Rev. bras. Geriatria e Gerontologia, 2015; 18(2): 239-248.

22. SOARES DS, et al. Fraturas de fêmur em idosos no Brasil: análise espaço-temporal de 2008 a 2012. Cad. Saúde Pública, Rio de Janeiro, 2014; 30(12): 2669-2678.

23. SOUZA FJM, et al. Percepção dos idosos institucionalizados acerca da qualidade de vida. Revista Eletrônica Acervo Saúde (REAS), 2020; 12(7): e3310.

24. WORLD HEALTH ORGANIZATION. WHO. Mental health of older adults. Geneva: 2017. Acesso em 19 de outubro de 2020. Disponível em: <https://www.who.int/news-room/fact-sheets/detail/mental-health-of-older-adults> 